\title{
Can neuroimaging inform economic theories of decision making?
}

\author{
This article was published in the following Dove Press journal: \\ Neuroscience and Neuroeconomics \\ 21 January 2013 \\ Number of times this article has been viewed
}

\author{
Norman AS Farb \\ Rotman Research Institute, Baycrest, \\ Toronto, Ontario, Canada
}

\begin{abstract}
Neuroimaging has grown in prominence with the popularization of relatively inexpensive and noninvasive techniques. The popular conception of neuroimaging is that it can finely describe a person's internal states and proclivities, providing veridical evidence in real-world situations, such as criminal trials, or in predicting consumer behavior. However, the scientific reality is far from this ideal; current neuroimaging techniques lack the precision to predict specific behaviors or preferences. Nonetheless, these techniques still possess considerable utility in describing forms of cognition that are recruited during decision making, such as an individual's tendency to focus on risk or reward. Such investigations can inform economic theory by characterizing contextual influences in decision making, revealing sources of bias in how value is appraised, generating new research hypotheses, and eventually leading to a more complete theory of human behavior. This review paper summarizes recent research that advances our understanding of the neural networks underlying decision making and outlines the strengths and limitations of current neuroimaging analysis techniques for informing neuroeconomic theory.
\end{abstract}

Keywords: neuroimaging, fMRI, decision making, neuroeconomics, systems neuroscience

\section{Introduction}

Functional neuroimaging attempts to relate brain activity to cognition and behavior. In the past decade, the emergence of more widespread access to neuroimaging technologies and analytic methods has led to the advent of neuroeconomics, a discipline dedicated to uncovering the myriad perceptual and motivational mechanisms underlying consumer choice. ${ }^{1,2}$ Generally employing "game-like" decision-making scenarios, researchers have attempted to integrate neural, physiological, and qualitative data into a single complex model of decision making. In recent years such research has advanced our understanding of how the brain represents several processes critical to economic decisions, such as the expectation of reward, ${ }^{3}$ the attribution of preference or avoidance attitudes, ${ }^{4}$ and the weighing of future utility against short-term hedonic gratification. ${ }^{5}$ A major strength of this approach is derived from economics' focus on relating brain activity to mathematically modeled behavioral predictions, ${ }^{3,6}$ yielding strong hypotheses for the detection of related neural activity. Yet while it is clear that economic paradigms have been successfully translated into neuroimaging domains, it is less clear how such research reciprocally informs economic theory.

This paper reviews whether current neuroimaging techniques can inform economically relevant cognition and behavior, beginning by addressing skeptical views of neuroeconomics' potential to contribute to economics, a science concerned more with the prediction of behavior than underlying cognitive processes, and arguing that some 
of these criticisms are justified: it is unlikely that this nascent field will soon aid in the measurement of consumer behavior as functional brain activity has not yet been shown to reliably predict individual decisions. However, neuroeconomics does not need to predict individual choices to have utility; its potential lies in broadening the scope of economic inquiry, identifying mechanisms of decision making that generate novel empirical hypotheses. In this respect neuroimaging has already made progress by identifying distinct neural connectivity systems associated with decision making, each responsible for a different information process. The association of particular information processing networks with a given decision-making context has the potential to inform new theoretical models and empirical studies. Following a discussion of how such contextual modeling can substantively contribute to economic theory, the paper concludes with a discussion of the potential future directions and potential of the field.

\section{It's economics - why bother with brains?}

While the "hows" and "whys" of decision making are of great interest to psychologists and philosophers, economists are more interested with outcomes, ie, "what" decision will be made within a given context. Critics of the neuroeconomic movement argue that since economic decisions are already observable through behavior, there is nothing to gain by studying brain mechanisms. ${ }^{7}$ For example, if a person chose product A over product B, why does it matter what parts of the brain were active? The traditional economist can argue that all the important information is already available - the product characteristics and the consumer choice - so why not simply "cut out the middle man" and rely upon behavioral experiments to study the factors influencing economic decisions?

Three responses are readily available to address questions of neuroimaging's utility. First, neuroimaging may offer greater fidelity than measures relying upon selfreport. In an experimental context, demand characteristics and participants' knowledge of their own evaluation may sometimes mask true response patterns. By measuring brain activity, researchers hope to bypass the problems inherent to conventional market testing, such as bias in focus groups or a lack of consumer insight into the true mechanisms of choice; such insight can inform both product development and subsequent marketing strategies. ${ }^{8}$ From an observational perspective, neuroimaging provides a unique source of information that may be relatively free from bias or demand characteristics.
The second response is that there are substantial information processes represented in the brain that determine economic decision making that are not easily observed through behavioral research methods. Recent experiments using transmagnetic stimulation to modulate brain activity demonstrate a causal connection between such stimulation and a person's valuation of goods, levels of self-interest, and even willingness to cheat trading partners. ${ }^{9}$ These studies confirm a causal relationship between brain activity and economic choice, above and beyond environmental factors, that merits investigation in a complete model of human behavior. So from an epistemological perspective, neuroimaging can provide access to cognitive processes determining economic behavior that are only the subject of conjecture in strictly behavioral approaches.

The third response is that neuroimaging has potential to discover new and important factors that contribute to economic theory. By characterizing and distinguishing between information processing pathways in the brain, neuroimaging may reveal fundamental principles of decision making that may not have been considered relevant in traditional economic research. For example, imaging techniques may help to explain how decision making changes under particular contexts, such as physical environment, mood, or a person's cultural background. Such contexts may transcend accounts of universally rational actors to explain why different people perceive value differently. From a scientific perspective, the modeling of how these different contextual factors influence decision making is important for making realistic predictions of behavior.

For example, let us consider a recent study that examined the effects of sleep deprivation on economic decision making. ${ }^{10}$ Following a night of sleep deprivation, participants were more likely to make risky decisions promoting low frequency rewards rather than maximizing the consistency of rewards. This behavioral finding may be interesting but is of little value to most firms, who cannot induce consumer sleep deprivation. On the other hand, knowing how sleep deprivation creates a perceptual context where reward immediacy is valued more than reward consistency might be of great value and generalizability. Firms could use such information to maximize the perception of value in marketing their products either by trying to shape the context in which the audience perceives the message or by predicting the audience's context and shaping the message to emphasize short-term gains or long-term consistency as is appropriate. The neural mechanisms of this sleep-deprivation effect and their economic implications will be discussed further below following a brief review of the neural circuits involved. 


\section{Neural circuitry of decision making}

An understanding of the basic neuroscientific theory underlying decision making is relevant to make these discussions of information processes concrete. This discussion focuses on the brain networks that seem critical to decision making and the evaluation of value, detailing how they work together to represent value, determine habitual behavior, and modify such habits to adaptively to account for "bottom-up" motivational relevance and "top-down" regulatory control. Over the past decade, there have been substantial advances in our understanding of these intrinsically connected brain networks. ${ }^{11}$ These networks were first identified by the reliable correlation between their constituent components, specific parts of the brain whose activation levels tend to move together. Since then, progress has been made in relating these networks to particular types of cognitive processes. Complex decision making is no longer thought to be represented by activity in a particular brain area. Instead, decision making is more likely a distributed process influenced by multiple networks representing competing contextual influences. ${ }^{9}$ Understanding the types of factors that compete to represent decision value can therefore benefit from a review of the major networks associated with decision making.

\section{The reward network}

The reward network is chief among the neural systems thought to drive decision making. The reward network is characterized as the primary system for the integration and representation of value information, chiefly characterized of interactions between the striatum and the prefrontal cortex (PFC). The distinction between these two broad regions is one of conditioned reward responses in the striatum and conceptual evaluation of value in the PFC. For example, one classic study documented that anticipation of gains in a reward-learning paradigm activates a region within the striatum known as the nucleus accumbens (NAc), and the magnitude of NAc activity correlates with reported excitement at the prospect of winning. ${ }^{12}$ Thus, the NAc signal represents the conditioned association between stimulus and reward, including the feelings of pleasure associated with that reward. In the same study the neural regions that responded to reward or loss outcomes rather than expectations were coded in the medial PFC (MPFC). The MFPC signal may represent the online evaluation of information, deriving reward value from the many properties that are present in a given attentional object. Supporting this view, MPFC activation has been associated with greater product preference across a wide variety of targets, including cars, ${ }^{13}$ beverages,,${ }^{14,15}$ and food. ${ }^{16}$

Extending the reward network theory, the ventral PFC bridging region known as the orbitofrontal cortex (OFC) is anatomically poised to integrate MPFC preference knowledge with striatum value prediction. The OFC appears to hold online the conceptual value of an attended object or action, acting as a value "accountant."17 Prior to its focus in neuroeconomic research, the OFC was hypothesized to act as a "somatic

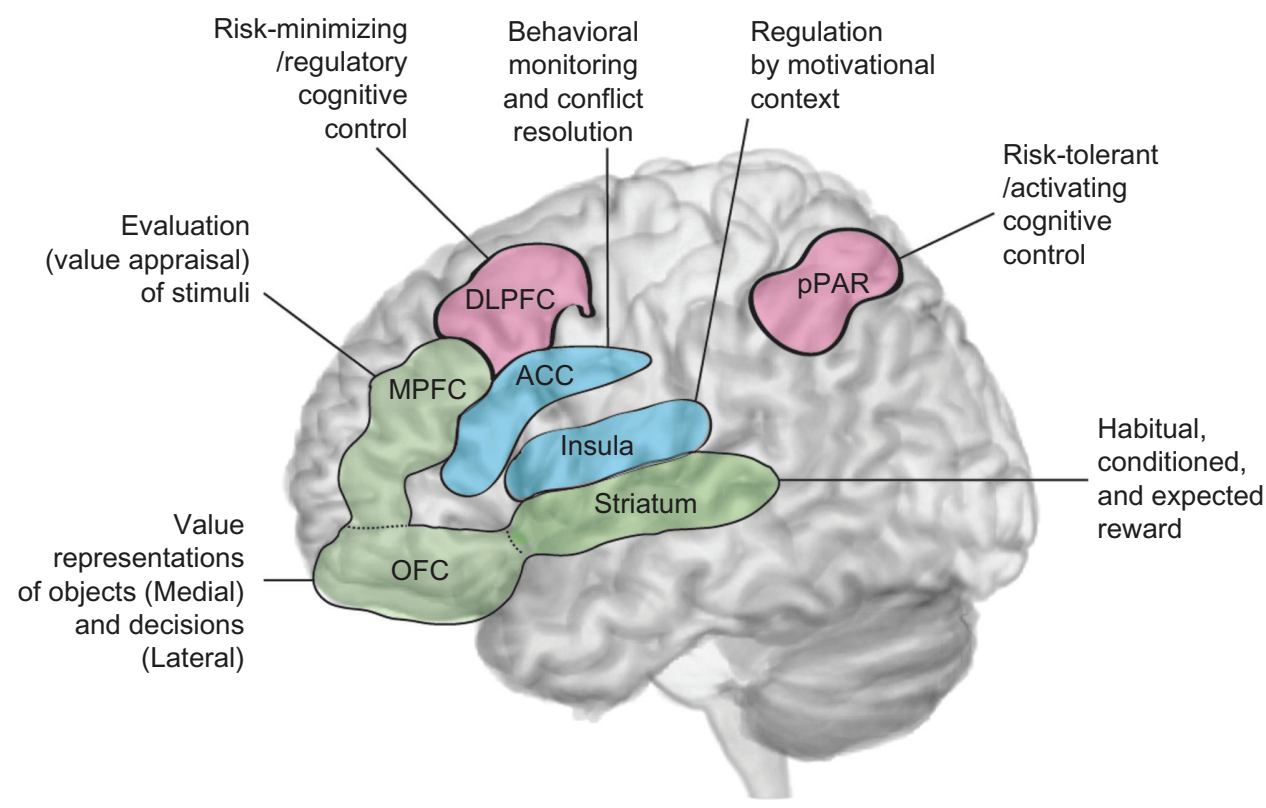

Figure I A summary of the three major networks associated with decision making.

Note: The reward network is shown in green, the salience network in blue, and the executive network in violet.

Abbreviations: ACC, anterior cingulate; DLPFC, dorsolateral prefrontal cortex; MPFC, medial prefrontal cortex; OFC, orbitofrontal cortex; pPAR, posterior parietal cortex. 
marker" region, ${ }^{18}$ serving as a cognitive proxy for visceral rewarding and aversive reactions to attentional objects. The somatic marker allows for rapid simulation of evaluative responses to imagined outcomes rather than requiring a physiological response from the body. In this way the OFC represents a region of abstract value information, distinct from behavioral associations or personal relevance. Empirical research has successfully characterized the OFC as coding for differences between the perceived value of goods, rather than subsequent choices that are instead linked to the dorsal striatum and supplementary motor cortex. ${ }^{19}$

In recent years the theory of OFC value representation has grown in complexity. Emerging research suggests considerable heterogeneity within the OFC regarding how specific types of value are represented. For instance, object values have been associated with the medial $\mathrm{OFC}^{20}$ compared to decision values with more lateral OFC and reward integration within the NAc. ${ }^{21}$ Additional distinctions have emerged along the OFC's anterior-posterior axis, linking object value to the anterior OFC and decision value to more posterior $\mathrm{OFC}$ nearer to the rewardintegrating striatum. ${ }^{22}$ Such findings suggest that different parts of the OFC may relate to different types of value calculations. In a study by Smith et al, ${ }^{22}$ more abstract financial reward value was represented in more anterior prefrontal regions than social rewards such as attractiveness, which had more direct impact on striatum activity, suggesting that physical distance from the striatum may be associated with the abstractness in value of the object under consideration. The idea of more anterior OFC performing more abstract value coding is supported by another study in which participants' more posterior OFC regions tracked actual reward outcomes from a chosen target, while the more anterior frontopolar regions represented the value of other targets that could have been chosen, ${ }^{23}$ indicating potential rather than actually obtained values. Such distinctions could be used to understand whether a product is being evaluated for its concrete, immediate value or for its more conceptual, longitudinal benefits. These OFC distinctions can also be used to explore individual differences in the tendency to think about broader abstract versus more concrete hedonic values to motivate behavior.

While dissociations between reward network regions are informative, it is important to remember that the network hypothesis is so named because individual network regions do not operate in isolation. In making decisions, the striatum and PFC are often coactivated, regardless of whether the decision is visceral or based on a more strategic analysis of the consequences of one's decisions. ${ }^{24}$ The striatum and PFC are also recruited under both estimable risks and unpredictable decision-making situations, ${ }^{25}$ so activation of this network is not constrained by factual evidence of reward but perhaps by imagined value as well. The finding that reward network activity may extend to imagined cues suggests that the interpretive context that an individual brings to the decision-making process may powerfully modulate the tuning of this network. Simply put, context determines what is considered rewarding. This idea is borne out in several recent studies in which health cues activated conceptual regulatory regions in the dorsolateral prefrontal cortex and altered the reward representations of different foods in the OFC, shifting reward-related activity to healthier rather than more appetitive food choices. ${ }^{16,26}$

Although there are many contextual variables to include when modeling the inputs to reward network activity, one encouraging finding in neuroeconomic research is that explicit consumer choice is not required for the neural representation of value.$^{27}$ Critically, neural responses to even unattended products appear to predict subsequent consumer choices, ${ }^{28}$ suggesting that the encoding of value in the brain may be habitual and automatic. If such findings can be refined to more accurately predict consumer choice, it will be possible to bypass many cognitive biases borne from social desirability or poor participant insight, directly mapping the building blocks of valuation that determine behavior.

\section{The need for multiple networks}

While the characterization of reward networks informs our understanding of value appraisal in decision making, consideration of contextual variables that frame these appraisals are needed to account for the breadth and flexibility of human motivation (Figure 1). Modeling motivational context requires understanding the regulation of the reward network, which may require the recruitment of a broader expanse of cortical and subcortical regions. ${ }^{29}$ For example, the general property of NAc activity to increase with anticipated rewards and decrease with anticipated losses only holds for certain outcomes. When uncertainty is involved, the magnitude of potential gains and losses predicts NAc activity. ${ }^{30}$ This finding supports the notion that under conditions of uncertainty or ambivalence, the NAc may represent a general motivational salience indicator that prompts involvement of broader prefrontal cortical networks. The multifaceted role for the NAc in representing reward has led many researchers to postulate that human decision making must be based upon multiple interacting neural systems. ${ }^{31,32}$

\section{The salience network}

Just as individual regions of the reward network do not operate in isolation, it is also the case that the reward network is just one of many competing networks for integration of environmental information and the direction of behavior. 
A second major network, which relies upon the insula and anterior cingulate, has been argued to represent the motivational salience of events. ${ }^{33}$ Anterior insula activity appears to track emotionally salient, and particularly aversive, events, ${ }^{34-36}$ although more recent evidence suggests that the anterior insula may represent anticipation of a contextually salient stimulus regardless of valence. ${ }^{37}$ Increasingly, the salience network appears to be important for adaptively integrating social and environmental cues in order to make appropriate choices; clinical disorders associated with poor social and economic decision making are associated with disruptions to salience network integrity. ${ }^{38}$ While dysfunction of the salience network is unlikely to be as pronounced in healthy individuals, activity in this network has the potential to reveal individual differences in relevant decision-making capacities, such as risk sensitivity and the ability to predict social and emotional consequences to behavior.

When studied together, both the reward and salience networks have important roles to play in determining individuals' choices. Whereas the reward network represents the value or utility of the choice, salience relates to how easily that choice captures attention and generates arousal. Together, the coactivation of these networks may powerfully motivate action. The interplay between these networks has begun to be explored experimentally, wherein both valuation and saliency have been shown to drive activation in the ventral striatum. Despite this common activation, value attribution appears to uniquely recruit the PFC and rostral and posterior cingulate cortex, while salience attribution appears to uniquely recruit the dorsal anterior cingulate, supplementary motor area, insula, and motor cortex. ${ }^{39}$ Thus, an additional contribution of current neuroeconomic research to contemporary decision-making theory is that both valuation and saliency properties are important considerations in understanding human behavior rather than appealing to a unitary construct of value alone. One role for these two networks may be to compete to determine the style by which a person pursues reward. In one reward-learning paradigm, the salience network was activated more strongly when switching to risk-avoidant strategies, whereas NAc activity was better associated with switching to risk-seeking strategies. ${ }^{40}$ The explanation for these networks is not as simple as pitting approach against aversion but one of adaptive behavior regulation over time. More immediate gratification choices are associated with reward network activation, whereas selecting longer delays over shorter delays is associated with salience network activity. ${ }^{41}$

The finding that a longstanding research paradigm, such as temporal discounting, may be driven by distinct networks for reward and motivation calculation is important for economic theory because it implies that decision making may be impacted in two fundamentally distinct ways. Increasing, the perceived value of a reward (irrespective of its temporal availability) should actually serve to promote the short-term gratification system, whereas increasing considerations of motivation and personal benefit should activate the longterm salience network. The generation of testable hypotheses for informing economic theory is one major benefit of the neuroimaging approach.

\section{The executive network}

One might be surprised by this description of the two major networks in decision making because neither of these networks is particularly linked to rational economics, featuring conceptual analysis or logical comparison of decision features and outcomes. The prevalence of habitual reward and emotional motivation networks in determining decisions is perhaps disconcerting to economists who wish to start from an axiom of rationality in predicting human behavior. On the other hand, this is not to say that reason and cognitive judgment are not also called into play when given the appropriate context. In ambiguous situations where reward values have not already been associated with a stimulus or behavior, a great deal of cognitive evaluation occurs, recruiting a network of brain regions chiefly characterized by the lateral PFC, which activates most strongly for participants who are risk averse. ${ }^{42}$ Conversely, for participants who prefer risk, posterior parietal cortex activation has been observed. These functionally connected frontoparietal regions have been implicated in a variety of tasks of cognitive control or the voluntary direction of attention, ${ }^{33,43}$ leading to their designation as the brain's executive network.

These lateral cortical regions are critical for top-down regulation of value appraisal, such as the example discussed above of attempting to eat what is healthy instead of just what tastes good. ${ }^{26}$ In this study, it was the influence of the executive network that was argued to modulate reward network responsiveness. Examining the conditions that both support and undermine executive network control over reward and salience network processing may be critical for understanding how and when seemingly rational actors fall victim to habit or emotional persuasion and conversely may also help to determine how best to strengthen the influence of reason in effecting change in consumer behavior.

\section{Additional networks}

In addition to the reward, salience, and executive networks, there are likely many more neural systems relevant to decision making that have yet to be well-characterized. For example, 
the choice of exactly how to act to achieve a selected object or goal may involve a third network for the "how" of achieving goals, recruiting a combination of supplementary motor areas and inferior parietal sulcus. ${ }^{44}$ Extending beyond even networks for physical action, real-world context often constrains decision making in unexpected ways that cannot be captured with the networks discussed so far. For instance, practical considerations often take precedence over even the most refined calculus between alternatives, such as how to get to the desired object, whether it can be transported safely, and whether it is socially permissible to attempt to acquire it. Identifying how such networks represent contextual information and serve to reinforce or constrain behavior may further inform economic theory and help to generate testable research hypotheses.

One example of a well-established network whose role in decision making is likely important but unclear is the default mode network (DMN). The DMN consists of a set of correlated brain regions that are deactivated by attentionally demanding tasks. ${ }^{45}$ Comprised of the posterior cingulate cortex, percuenus, ventromedial PFC, medial temporal lobe, and inferior parietal cortex, the DMN has not been directly linked to economic decision making, although the ability to deactivate the DMN appears very important for the efficient direction of attention. In patients with traumatic brain injury, for example, DMN hyperactivity predicts attention deficits. ${ }^{46}$ The salience network appears to be particularly important for disengaging the DMN when attention-demanding decisions are required; degradation of salience network white matter predicts failures in DMN disengagement following traumatic brain injury. ${ }^{47}$ The specific function of the DMN is still under debate, but its deactivation could potentially be used as a fruitful index of a person's attentional engagement in the context of a decisionmaking task. Conversely, high levels of DMN activity have been linked to mind wandering ${ }^{48}$ and conceptually elaborated self-referential thought. ${ }^{49} \mathrm{DMN}$ activity may therefore indicate a self-centered rather than task-centered focus; it has also been theorized that disruptions to DMN activity may disrupt assessments of an object or a decision's self-relevance, ${ }^{50}$ leading to poor goal setting in unconstrained decision-making contexts. More specific research will be required to demonstrate the applicability of these theories to economic decision making. The DMN does, however, provide the promise of integrating higher order goals and values into a decision-making context.

\section{Generating theories of contextually bounded decision making}

Armed with this growing understanding of neural systems supporting decision making, we may return to the earlier example of sleep deprivation changing the criteria for value, which contained the acquisition of neuroimaging data to complement its behavioral findings. ${ }^{10}$ Following sleep deprivation, participants demonstrated reduced anterior insula activation and increased ventromedial PFC activation; the magnitude of these changes then predicted the tendency to make risky decisions. Ventromedial PFC damage appears to generally impair consistency in decision making, regardless of the certainty of decision outcomes. ${ }^{51}$ Increased activity in this region following sleep deprivation may therefore reflect greater difficulty in stably representing alternative decision values following sleep deprivation. By considering brain network evidence, a greater context is given to the study's behavioral findings. Sleep deprivation appears to increase sensitivity to immediate gains by decreasing processing efficiency in the reward network, while reducing sensitivity to potential losses in the motivational salience network.

In the sleep deprivation study, the increased endorsement of short-term reward following sleep deprivation was observable from behavioral research alone. ${ }^{51}$ However, the broader implication, that there may be two competing systems for managing risk and reward in the brain, changes the paradigm for marketing and product development. In designing a product and subsequent marketing, determining which of these systems will be relied upon to determine a product's value is an important consideration. Questions of personality are ultimately addressable through behavioral research, such as measuring whether risk-averse or riskseeking individuals are more likely to endorse a given product or advertisement. However, the generation of these research hypotheses stems from the neuroimaging finding of a link between sleep deprivation and two competing brain systems managing risk tolerance, an insight that is unlikely to have arisen from the behavioral evidence alone.

In addition to changing the way that firms design and market their products, neuroimaging also has the potential to advance decision-making theory and fuel further empirical research. In another study researchers asked participants to choose a target product when confronted with pairs of similar product brands. ${ }^{52}$ High levels of executive network activity were observable during decision making of branded products when the target was not the participant's preferred brand. On the other hand, when the target was the participant's favorite brand, participants showed reduced executive network activity but increased activation of the reward network, suggesting that selecting one's preferred brand had become a habitual emotionally driven form of decision making rather than cognitive comparison or elaboration. Such results speak 
directly toward a divergent economic theory for maintaining brand loyalty versus attracting new customers; maintenance of brand loyalty may be more likely in situations when a person does not engage in conceptual evaluative thinking, whereas switching to a nonpreferred brand may be more likely to occur when a person is put into an analytic mode, weighing the pros and cons of their purchasing decisions. In this way, the neuroimaging findings move beyond a purely rational description of the actor to describe the mental context in which different types of decisions will be made. Whether or not such hypotheses will bear fruit is an empirical question, one that stems from an analysis of the neuroimaging data. While undoubtedly economists take their research inspiration from a variety of sources, this does not discount the value of looking into neuroeconomic approaches as another source of inspiration.

\section{What about predicting specific decisions?}

Despite potential theoretical applications afforded by a growing understanding of the basic mechanisms of choice, for many the true allure of neuroimaging lies in predicting specific decisions. One promising field of research in predicting human behavior from brain activity involves machine learning algorithms that learn to classify brain activity patterns to predict specific decisions. Thus far, pattern classification techniques have shown that brain information can improve prediction of what type of visual stimulus is being observed, ${ }^{53,54}$ remembered, ${ }^{55}$ or in distinguishing between specific gestures. ${ }^{44}$ These pattern-classification techniques, most notably multi-voxel pattern analysis, have the advantage of searching through the brain in an unbiased way to provide the best evidence of a distinction based on neural activity information. ${ }^{56}$ On the other hand, it is important not to lose sight of the fact that in almost all cases, pattern classification accuracy is only modestly greater than chance, ${ }^{44,53-55,57,58}$ it is unlikely that marketers and firms will want to trust a prediction algorithm that is only $60 \%$ accurate in predicting client preferences, no matter how statistically significant this prediction ability happens to be.

The limitations of current techniques are apparent when one considers even the recent sophisticated neuroimaging research efforts. For example, one recent study incorporated insights about competing reward and salience networks and attempted to integrate this information in a predictive model of decision making. ${ }^{58}$ In this study, it was simultaneously demonstrated that object preference was encoded by the NAc, value disparity between willingness to pay and price was encoded in the MPFC, and purchasing was correlated with bilaterally deactivated salience network hubs in the insula. All of these observations were used in a pattern classification analysis to attempt to improve upon participants' reported preferences, a much easier measure to collect. Reported preferences could account for $53 \%$ of purchase decisions. Brain activation patterns did account for purchases above and beyond self-reported preferences but only improved prediction of purchases by $0.05 \%$. Another recent study looked at simplified decision learning between two arbitrary stimuli and was able to show greater performance, around $60 \%$ accuracy. ${ }^{57}$ While additional decision-making information appears to exist within the brain signal, our current ability to extract it to make a practical difference is clearly still limited.

On the other hand, these first fledgling steps need not promote a sense of despondence and pessimism. Temporal and spatial resolution in neuroimaging will likely improve over time, and even now there are many things that we can do to improve predictive efficacy. One important factor to consider when attempting to use neural activity to predict behavior is that people differ in their motivational context and these differences may add considerable noise to the data. Take for example the idea that people vary greatly in their risk-taking tendencies. In one study, ${ }^{59}$ dorsal MPFC activity was recruited when participants engaged in risky decisions, but the magnitude of this activation was predicted by risk aversion. However, ventral MPFC activity was modulated by actual gains, and the magnitude of this modulation was correlated with higher levels of risk tolerance. Thus, for someone strongly risk avoidant, dorsal prefrontal regions associated with cognitive control and vigilance are likely to serve as an indicator of a cautious willingness to take risks. For someone who is use to taking risk, however, such monitoring activity is less likely to be diagnostic of a risky decision; instead the magnitude of the ventral reward signal itself is a better predictor of behavior. If these important individual differences in personality can be identified and validated, there is every indication that knowing how to model brain activity for different types of people will improve predictive power.

\section{The limits of functional magnetic resonance imaging}

While this review supports the potential for functional magnetic resonance imaging (fMRI) to inform our understanding of functional brain mechanisms, it is important to remember that the technology still has many limitations. While fMRI 
allows us to look at broad patterns of brain activity using blood deoxygenation as a proxy for metabolism, not all information about brain function is reflected in such gross measures. ${ }^{60}$ For example, a measurement of hand metabolism may give insight into the difference between working as a concert piano player or construction worker; a construction worker most likely shows broader coordinated muscle contraction, whereas the piano player shows smaller rapid activation in fingers, perhaps with an underlying rhythmic frequency. So from this perspective we can learn a lot about the differences between the two professions from studying hand metabolism. On the other hand, such an approach is very limited; it would be difficult using hand metabolism to appreciate differences in pieces of music or to predict what notes the piano player is playing; similarly, it would be almost impossible to predict what sort of building the construction worker is working on. Second, there is also a problem of temporal resolution in fMRI; notes played quickly would not be tracked as fMRI only takes whole brain pictures once every $2-3$ seconds, so rapid communication or change is not available and such information is lost to us using current technology. Third, while fMRI has good spatial resolution for looking at $2 \mathrm{~mm}$ brain volumes, tracking specific neurons is beyond our current ability, so nuanced local network communication is not observable. Fourth, looking at specific neurochemicals with fMRI is not well developed; in theory it is possible through MR spectroscopy, but such practices are not widespread and are generally confined to a small part of the brain in a given study. The limitations notwithstanding, there is still much to be gained in understanding the dynamics of broad brain networks, even as more specific and nuanced analytic technologies are being developed.

\section{Conclusion}

This paper has laid out some of the potential benefits for the use of neuroimaging in neuroeconomic research. In the short term, the utility of neuroimaging in advancing economic theory probably does not lie in its ability to stand as a proxy for measurable consumer behaviors, at least not until neuroimaging becomes as cheap and direct as behavioral research. Furthermore, as discussed in the final section on predicting specific decisions, it is not realistic to expect current neuroimaging techniques to forecast behavior with high levels of predictive accuracy. On the other hand, considerable value has already been demonstrated in studying how the brain represents value, revealing the tension between rational evaluation and habit in dictating many of our decisions. Even for economists well versed in the power of consumer habits, new insights and hypotheses are available from emerging research, such as how motivational and regulatory influences are represented in distinct neural networks that constrain the appraisal process. Confronted with tangible evidence that motivational relevance is separately and competitively encoded in the brain, economists may experiment with models in which such motivational mindsets are determined, leading to more complete theories of human behavior. Exactly how such investigations will unfold and contribute to economic theory over the next decade is difficult to predict, but it seems likely that neuroeconomics will continue to inform and challenge the traditional limits of economic theory to more broadly account for the contextual influences guiding human behavior.

\section{Disclosure}

The author reports no conflicts of interest in this work.

\section{References}

1. Glimcher PW, Rustichini A. Neuroeconomics: the consilience of brain and decision. Science. 2004;306(5695):447-452.

2. Montague PR, Berns GS. Neural economics and the biological substrates of valuation. Neuron. 2002;36(2):265-284.

3. Kishida KT, Montague PR. Imaging models of valuation during social interaction in humans. Biol Psychiatry. 2012;72(2):93-100.

4. O'Doherty JP. Reward representations and reward-related learning in the human brain: insights from neuroimaging. Curr Opin Neurobiol. 2004;14(6):769-776.

5. Hare TA, Camerer CF, Rangel A. Self-control in decision-making involves modulation of the vmPFC valuation system. Science. 2009; 324(5927):646-648.

6. Montague PR, King-Casas B, Cohen JD. Imaging valuation models in human choice. Annu Rev Neurosci. 2006;29:417-448.

7. Bernheim BD. The psychology and neurobiology of judgment and decision making: What's in it for economists? In: Glimcher PW, Camerer CF, Fehr E, Poldrack RA, editors. Neuroeconomics: Decision Making and the Brain. London: Elsevier; 2009:115-125.

8. Ariely D, Berns GS. Neuromarketing: the hope and hype of neuroimaging in business. Nat Rev Neurosci. 2010;11(4):284-292.

9. Fehr E, Rangel A. Neuroeconomic Foundations of Economic Choice - Recent Advances. J Econ Perspect. 2011;25(4):3-30.

10. Venkatraman V, Huettel SA, Chuah LY, Payne JW, Chee MW. Sleep deprivation biases the neural mechanisms underlying economic preferences. J Neurosci. 2011;31(10):3712-3718.

11. Fox MD, Raichle ME. Spontaneous fluctuations in brain activity observed with functional magnetic resonance imaging. Nat Rev Neurosci. 2007;8(9):700-711.

12. Knutson B, Fong GW, Adams CM, Varner JL, Hommer D. Dissociation of reward anticipation and outcome with event-related fMRI. Neuroreport. 2001;12(17):3683-3687.

13. Erk S, Spitzer M, Wunderlich AP, Galley L, Walter H. Cultural objects modulate reward circuitry. Neuroreport. 2002;13(18):2499-2503.

14. Paulus MP, Frank LR. Ventromedial prefrontal cortex activation is critical for preference judgments. Neuroreport. 2003;14(10): 1311-1315.

15. McClure SM, Li J, Tomlin D, Cypert KS, Montague LM, Montague PR. Neural correlates of behavioral preference for culturally familiar drinks. Neuron. 2004;44(2):379-387. 
16. Siep N, Roefs A, Roebroeck A, Havermans R, Bonte ML, Jansen A. Hunger is the best spice: an fMRI study of the effects of attention, hunger and calorie content on food reward processing in the amygdala and orbitofrontal cortex. Behav Brain Res. 2009;198(1):149-158.

17. Fellows LK. Advances in understanding ventromedial prefrontal function: the accountant joins the executive. Neurology. 2007;68(13):991-995.

18. Damasio AR. The somatic marker hypothesis and the possible functions of the prefrontal cortex. Philos Trans R Soc Lond B Biol Sci. 1996;351(1346):1413-1420.

19. FitzGerald TH, Seymour B, Dolan RJ. The role of human orbitofrontal cortex in value comparison for incommensurable objects. $J$ Neurosci. 2009;29(26):8388-8395.

20. Plassmann H, O’Doherty J, Rangel A. Orbitofrontal cortex encodes willingness to pay in everyday economic transactions. J Neurosci. 2007;27(37):9984-9988.

21. Hare TA, O’Doherty J, Camerer CF, Schultz W, Rangel A. Dissociating the role of the orbitofrontal cortex and the striatum in the computation of goal values and prediction errors. J Neurosci. 2008;28(22):5623-5630.

22. Smith DV, Hayden BY, Truong TK, Song AW, Platt ML, Huettel SA. Distinct value signals in anterior and posterior ventromedial prefrontal cortex. J Neurosci. 2010;30(7):2490-2495.

23. Boorman ED, Behrens TE, Woolrich MW, Rushworth MF. How green is the grass on the other side? Frontopolar cortex and the evidence in favor of alternative courses of action. Neuron. 2009;62(5):733-743.

24. Daw ND, Gershman SJ, Seymour B, Dayan P, Dolan RJ. Model-based influences on humans' choices and striatal prediction errors. Neuron. 2011;69(6):1204-1215.

25. Levy I, Snell J, Nelson AJ, Rustichini A, Glimcher PW. Neural representation of subjective value under risk and ambiguity. J Neurophysiol. 2010;103(2):1036-1047.

26. Hare TA, Malmaud J, Rangel A. Focusing attention on the health aspects of foods changes value signals in vmPFC and improves dietary choice. J Neurosci. 2011;31(30):11077-11087.

27. Levy I, Lazzaro SC, Rutledge RB, Glimcher PW. Choice from non-choice: predicting consumer preferences from blood oxygenation level-dependent signals obtained during passive viewing. J Neurosci. 2011;31(1):118-125.

28. Tusche A, Bode S, Haynes JD. Neural responses to unattended products predict later consumer choices. J Neurosci. 2010;30(23):8024-8031.

29. Rushworth MF, Behrens TE. Choice, uncertainty and value in prefrontal and cingulate cortex. Nat Neurosci. 2008;11(4):389-397.

30. Cooper JC, Knutson B. Valence and salience contribute to nucleus accumbens activation. Neuroimage. 2008;39(1):538-547.

31. Daw ND, Niv Y, Dayan P. Uncertainty-based competition between prefrontal and dorsolateral striatal systems for behavioral control. Nat Neurosci. 2005;8(12):1704-1711.

32. Rangel A, Camerer C, Montague PR. A framework for studying the neurobiology of value-based decision making. Nat Rev Neurosci. 2008;9(7):545-556.

33. Seeley WW, Menon V, Schatzberg AF, et al. Dissociable intrinsic connectivity networks for salience processing and executive control. J Neurosci. 2007;27(9):2349-2356.

34. Paulus MP, Stein MB. An insular view of anxiety. Biol Psychiatry. 2006;60(4):383-387.

35. Paulus MP, Rogalsky C, Simmons A, Feinstein JS, Stein MB. Increased activation in the right insula during risk-taking decision making is related to harm avoidance and neuroticism. Neuroimage. 2003;19(4):1439-1448.

36. Simmons A, Matthews SC, Stein MB, Paulus MP. Anticipation of emotionally aversive visual stimuli activates right insula. Neuroreport. 2004; 15(14):2261-2265.

37. Lovero KL, Simmons AN, Aron JL, Paulus MP. Anterior insular cortex anticipates impending stimulus significance. Neuroimage. 2009;45(3): 976-983.

38. Menon V. Large-scale brain networks and psychopathology: a unifying triple network model. Trends Cogn Sci. 2011;15(10):483-506.
39. Litt A, Plassmann H, Shiv B, Rangel A. Dissociating valuation and saliency signals during decision-making. Cereb Cortex. 2011;21(1): 95-102.

40. Kuhnen CM, Knutson B. The neural basis of financial risk taking. Neuron. 2005;47(5):763-770.

41. Wittmann M, Leland DS, Paulus MP. Time and decision making: differential contribution of the posterior insular cortex and the striatum during a delay discounting task. Exp Brain Res. 2007;179(4): 643-653.

42. Huettel SA, Stowe CJ, Gordon EM, Warner BT, Platt ML. Neural signatures of economic preferences for risk and ambiguity. Neuron. 2006;49(5):765-775.

43. Corbetta M. Frontoparietal cortical networks for directing attention and the eye to visual locations: identical, independent, or overlapping neural systems? Proc Natl Acad Sci US A. 1998;95(3):831-838.

44. Gallivan JP, McLean DA, Valyear KF, Pettypiece CE, Culham JC. Decoding action intentions from preparatory brain activity in human parieto-frontal networks. J Neurosci. 2011;31(26):9599-9610.

45. Raichle ME, MacLeod AM, Snyder AZ, Powers WJ, Gusnard DA, Shulman GL. A default mode of brain function. Proc Natl Acad Sci US A. 2001;98(2):676-682.

46. Bonnelle V, Leech R, Kinnunen KM, et al. Default mode network connectivity predicts sustained attention deficits after traumatic brain injury. J Neurosci. 2011;31(38):13442-13451.

47. Bonnelle V, Ham TE, Leech R, et al. Salience network integrity predicts default mode network function after traumatic brain injury. Proc Natl Acad Sci U S A. 2012;109(12):4690-4695.

48. Christoff K, Gordon AM, Smallwood J, Smith R, Schooler JW. Experience sampling during fMRI reveals default network and executive system contributions to mind wandering. Proc Natl Acad Sci USA. 2009;106(21):8719-8724.

49. Farb NA, Segal ZV, Mayberg H, et al. Attending to the present: mindfulness meditation reveals distinct neural modes of self-reference. Soc Cogn Affect Neurosci. 2007;2(4):313-322.

50. Sonuga-Barke EJ, Fairchild G. Neuroeconomics of attention-deficit/ hyperactivity disorder: differential influences of medial, dorsal, and ventral prefrontal brain networks on suboptimal decision making? Biol Psychiatry. 2012;72(2):126-133.

51. Fellows LK, Farah MJ. The role of ventromedial prefrontal cortex in decision making: judgment under uncertainty or judgment per se? Cereb Cortex. 2007;17(11):2669-2674.

52. Deppe M, Schwindt W, Kugel H, Plassmann H, Kenning P. Nonlinear responses within the medial prefrontal cortex reveal when specific implicit information influences economic decision making. J Neuroimaging. 2005;15(2):171-182.

53. Kamitani Y, Tong F. Decoding the visual and subjective contents of the human brain. Nat Neurosci. 2005;8(5):679-685.

54. Haxby JV, Gobbini MI, Furey ML, Ishai A, Schouten JL, Pietrini P. Distributed and overlapping representations of faces and objects in ventral temporal cortex. Science. 2001;293(5539):2425-2430.

55. Harrison SA, Tong F. Decoding reveals the contents of visual working memory in early visual areas. Nature. 2009;458(7238):632-635.

56. Norman KA, Polyn SM, Detre GJ, Haxby JV. Beyond mind-reading: multi-voxel pattern analysis of fMRI data. Trends Cogn Sci. 2006; 10(9):424-430.

57. Hampton AN, O'Doherty JP. Decoding the neural substrates of reward-related decision making with functional MRI. Proc Natl Acad Sci US A. 2007;104(4):1377-1382.

58. Knutson B, Rick S, Wimmer GE, Prelec D, Loewenstein G. Neural predictors of purchases. Neuron. 2007;53(1):147-156.

59. Xue G, Lu Z, Levin IP, Weller JA, Li X, Bechara A. Functional dissociations of risk and reward processing in the medial prefrontal cortex. Cereb Cortex. 2009;19(5):1019-1027.

60. Arthurs OJ, Boniface S. How well do we understand the neural origins of the fMRI BOLD signal? TRENDS in Neurosciences. 2002;25(1): 27-31. 


\section{Publish your work in this journal}

Neuroscience and Neuroeconomics is an international, peer-reviewed, open access journal focusing on the identification of brain structures and measurement of neural activity related to behavior, behavioral predictions, and decision making in health and disease. The manuscript management system is completely online and includes a very quick and

Submit your manuscript here: http://www.dovepress.com/neuroscience-and-neuroeconomics-journal fair peer-review system. Visit http://www.dovepress.com/testimonials. php to read real quotes from published authors. 